University of Nebraska - Lincoln

DigitalCommons@University of Nebraska - Lincoln

4-15-1987

\title{
Magnetic transitions and scaling in anisotropic rare-earth glasses
}

Kevin M. Lee

Kansas State University, klee6@unl.edu

Michael J. O'Shea

Kansas State University, mjoshea@phys.ksu.edu

David J. Sellmyer

University of Nebraska-Lincoln, dsellmyer@unl.edu

Follow this and additional works at: https://digitalcommons.unl.edu/physicssellmyer

Part of the Physics Commons

Lee, Kevin M.; O'Shea, Michael J.; and Sellmyer, David J., "Magnetic transitions and scaling in anisotropic rare-earth glasses" (1987). David Sellmyer Publications. 139.

https://digitalcommons.unl.edu/physicssellmyer/139

This Article is brought to you for free and open access by the Research Papers in Physics and Astronomy at DigitalCommons@University of Nebraska - Lincoln. It has been accepted for inclusion in David Sellmyer Publications by an authorized administrator of DigitalCommons@University of Nebraska - Lincoln. 


\title{
Magnetic transitions and scaling in anisotropic rare-earth glasses
}

\author{
K. M. Lee and M. J. O'Shea \\ Cardwell Hall, Department of Physics, Kansas State University, Manhattan, Kansas 66506 \\ D. J. Sellimyer \\ Behlen Laboratory of Physics, University of Nebraska, Lincoln, Nebraska 68588-0111
}

\begin{abstract}
We report studies of the magnetic critical behavior as a function of anisotropy strength in rareearth-rich glasses. Anisotropy is induced in Gd-Co by alloying with $\mathrm{Tb}$. The system studied is $\mathrm{Gd}_{65-x} \mathrm{~Tb}_{x} \mathrm{CO}_{35}$ and the random magnetic anisotropy (RMA) strength may be increased by increasing $x$. The $x=0$ alloy of this series has been shown to exhibit standard critical behavior expected for a second-order phase transition. With increasing $x$ we find deviations from this behavior due to the presence of RMA. We present detailed results for the alloy with composition $x=50$ at. \% and show that for large enough magnetic fields the system continues to show standard ferromagnetic scaling but for low enough fields the system crosses over to a behavior describabie by nonlinear sealing similar to that found in spin-glass systems.
\end{abstract}

We report on the transition behavior in rare-earth-rich glasses as a function of anisotropy strength and discuss the scaling behavior at the speromagnetic (SM) transition. The SM state has been discussed by Coey. ${ }^{1}$ In this state the spins are frozen in a random fashion by the strong random magnetic anisotropy (RMA) present.

The alloys of this work are based on the much studied $\mathrm{Gd}-\mathrm{Co}$ system and are of the form $\mathrm{Gd}_{65} \mathrm{~Tb}_{x} \mathrm{CO}_{35}$. The glasses are prepared by splat-cooling and are in the form of circular foils about $50 \mu \mathrm{m}$ in thickness. CuKa $\mathrm{x}$-rays were used to check the structure and no evidence for crystallinity was found. Magnetization measurements were made below room temperature as a function of applied field up to $80 \mathrm{kOe}$ using a vibrating sample magnetometer (VSM) equipped with a superconducting magnet and below 1 kOe using a VSM equipped with a wire wound coil. ac susceptibility measurements were made in an rms field of 10 at a frequency of $280 \mathrm{~Hz}$. Samples for both ac susceptibility and magnetization measurements were in the form of sandwiches of about eight strips, $1.0 \mathrm{~cm}$ in length and about $0.15 \mathrm{~cm}$ wide, with their long axes aligned parallel to the applied field to reduce demagnetization effects.

Amorphous $\mathrm{Gd}_{65} \mathrm{CO}_{35}$ is ferrimagnetic and the work of Fukamichi and co-workers ${ }^{2}$ shows that the $\mathrm{Gd}$ and Co ions carry moments of 6.9 and $0.6 \mu_{B}$, respectively.

The ac susceptibility for the Tb system of this work has already been reported. ${ }^{3}$ The alloy with composition $x=0$ shows a transition to a ferrimagnetic state at $186 \mathrm{~K}$. The reduction in susceptibility below the transition is probably due to the presence of a small amount of anisotropy in $\mathrm{Gd}_{65} \mathrm{Co}_{35}$. Alloying with $\mathrm{Tb}$ (increasing $x$ ) leads to a crossover to PM-SM behavior in ac susceptibility with only a small peak being present for large $x$. Figure 1 shows the transition temperatures as a function of $x$. The reduction in ordering temperature is due in part to a reduction in the average value of $s(s+1)$ ( $s$ represents spin) as one would expect for a system where the RKKY interaction is being varied. Similar behavior has been observed in crystalline intra-rare-earth compounds. ${ }^{4}$ The reduction of $s(s+1)$ on alloying with $\mathrm{Tb}(15.75$ to $12 \mathrm{in}$ going from $x=0$ to 65$)$ is not enough by itself to account for the lowering of the ordering temperature. Experiments on amorphous and crystalline $\mathrm{Tb}-\mathrm{Fe}$ (Ref.5) have already shown that a strong RMA leads to a lowering of transition temperature and we ascribe part of the reduction in transition temperature to increasing RMA strength. Magnetization measurements up to 75 kOe were made to study hysteresis and Fig. 2 shows hysteresis loops for some selected alloys. The coercivity as a function of composition $x$ was determined from magnetization measurements at $4.2 \mathrm{~K}$ and these results are shown in Fig. 1. As expected the coercive force increases with increasing Tb content due to the increase in anisotropy strength.

Previous workers have noted the experimental and theoretical connections between spin-glass and RMA systems. ${ }^{6}$ Generally both types of system exhibit similar magnetic states at low temperatures with the spins being frozen in random directions. In particular a scaling description of the PM-SM transition similar to that of the spin-glass transition has been shown to hold. ${ }^{7,8}$ According to this scaling

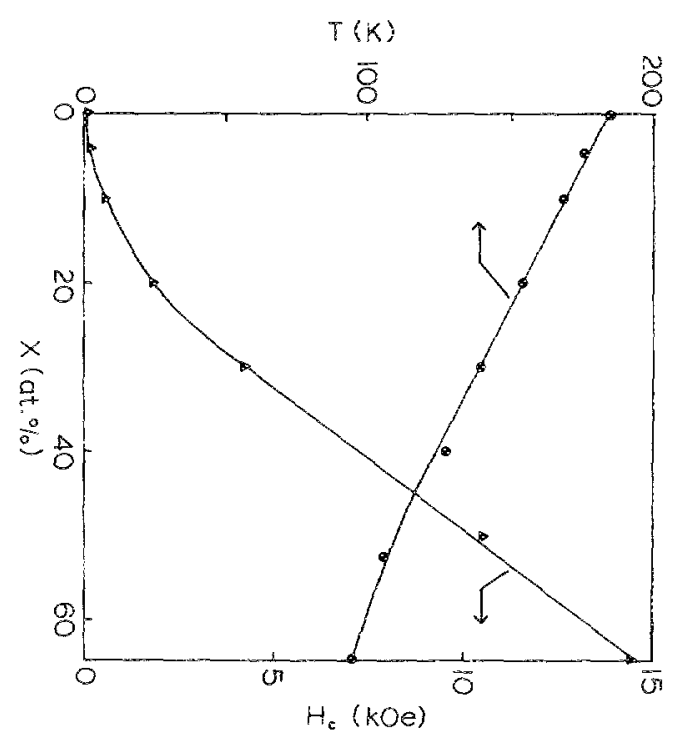

FIG. 1. Transition temperature and coercivity (at $4.2 \mathrm{~K}$ ) as a function of composition for the $\mathrm{Gd}_{65-x} \mathrm{~Tb}_{x} \mathrm{Co}_{35}$ system. 


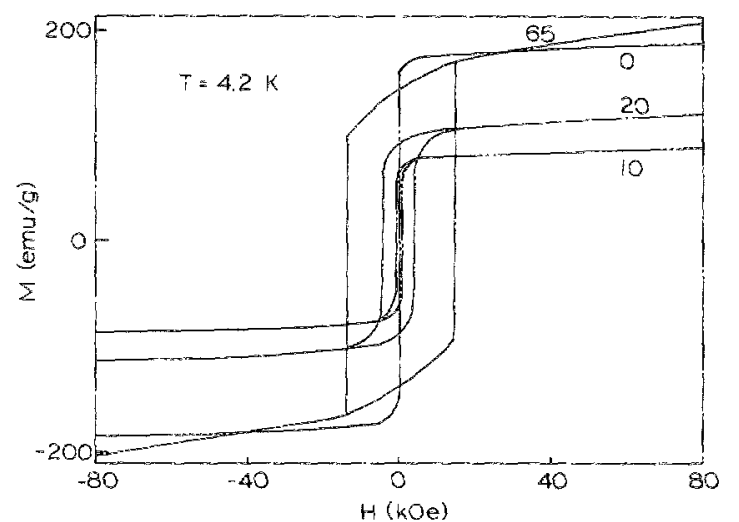

FIG. 2. Hysteresis loops for selected alloys at $4,2 \mathrm{~K}$. The coercivity increases from 10 oe for the $x=0$ sample to $14.3 \mathrm{kOe}$ for the $x=65$ sample.

hypothesis the general magnetic equation of state can be written in the form?

$$
m_{n 1}=f_{ \pm}\left(h_{n 1}\right) \text {. }
$$

where

$$
m_{n 1}=M_{n 1} / \epsilon^{(3 \beta+\gamma) / 2}, \quad h_{n 1}=H / \epsilon^{(\beta+\gamma) / 2} .
$$

$M_{n 1}(T)=\chi_{0}(T) H-M(T)$ is the nonlinear magnetization, $\epsilon=\left|\left(T-T_{c}\right) / T_{c}\right|$ is a reduced temperature and $\chi_{a}(T)$ is the zero-field susceptibility calculated from the initial slope of a magnetic isotherm taken at a giver tempera* ture $T$. The \pm sign corresponds to two branches of the equation of state, $T>T_{c}$ and $T<T_{c}$, respectively.

We have done a scaling study of the magnetization of the alloy with composition $x=50$ at. \% at low fields ( $<1 \mathrm{kOe}$ ) and at high felds (up to $80 \mathrm{kOe}$ ) to determine how well such a description holds in systems where RMA is important. The low-field susceptibility (both ac and dc) is about a factor of 20 below the demagnetization limit (Ref. 3, Fig. 1) and so demagnetization effects are not important in this system. We find that the magnetization data above 1 kOe do not follow the nonlinear scalling equation of state, Eq. (1) $(\beta$ and $\gamma$ were varied in the range $0.3-3$ and $1-10$, respectively), even though we have determined the transition temperature to within $0.2 \mathrm{~K}$ and the zero-field susceptibilities $\chi(T)$ required to compute the nonlinear susceptibility to about $2 \%$. We do find, however, that the high-field magnetization follows the standard ferromagnetic scaling behavior in this glass even though a strong RMA is present. The standard ferromagnetic scaling equation is similar to $\mathrm{Eq}$. (1) and is given by ${ }^{10}$

$$
m=g_{ \pm}(h)
$$

where

$$
m=M / \epsilon^{\beta}, \quad h=H / \epsilon^{\beta \delta} .
$$

Figure 3 shows the magnetization data for the glass with composition $x=50$ at. \% as a function of applied feld in both linear and logarithmic form to resolve the scaling at both high and lower fields. The exponents $\beta$ and $\delta$ of Eq. (2) were found to be $0.46 \pm 0.01$ and $4.0 \pm 0.1$, respectively. These values are close to those found in a number of other metallic glasses and to the Heisenberg $d=3$ model ${ }^{10}$ Below about 1 kOe large deviations (not shown) from scaling ac-
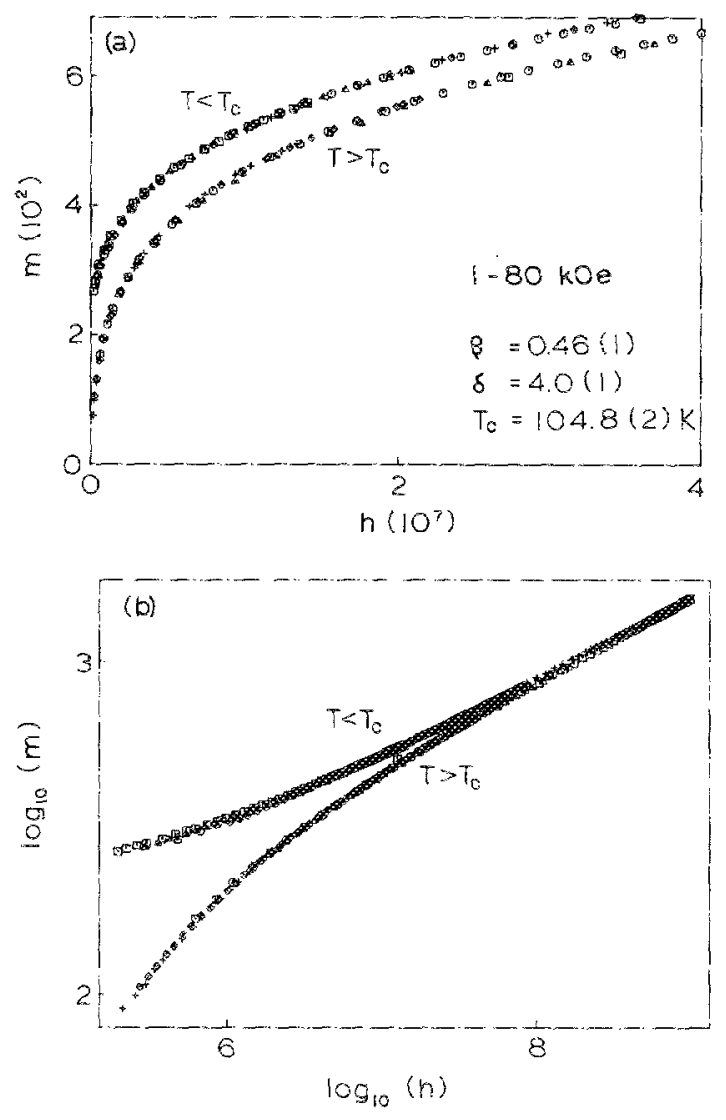

FIG. 3. Scaling of the magnetic isotherms for the $x=50$ giass plotted in (a) linear and (b) logarithmic form. $m$ and $h$ are reduced units as described in the text. The linear plot is cut off I decade below the logarithmic plot in reduced feld so that the scaling can be resolved at internediate fields. The sealing at the highest reduced units is of similar quality to that in panel (a). The temperatures of the magnetic isotherms in degrees Kelvin are 111.3 $(+), 109.8(0) 108.4(0), 106.8(\Delta), 105.4(0), 104.1(+), 102.4(0)$, $101.0(0), 99.7(\Delta), 98.5([])$.

cording to Eq. (2) become apparent. This should be contrasted with a number of other systems studied by us and others where good scaling is found down to 50 Oe and below with the low-field limit being determined by demagnetization field effects. ${ }^{10,11}$

Below 700 Oe we tested the magnetic isotherms to see how well they conform to a noninear scaling equation of

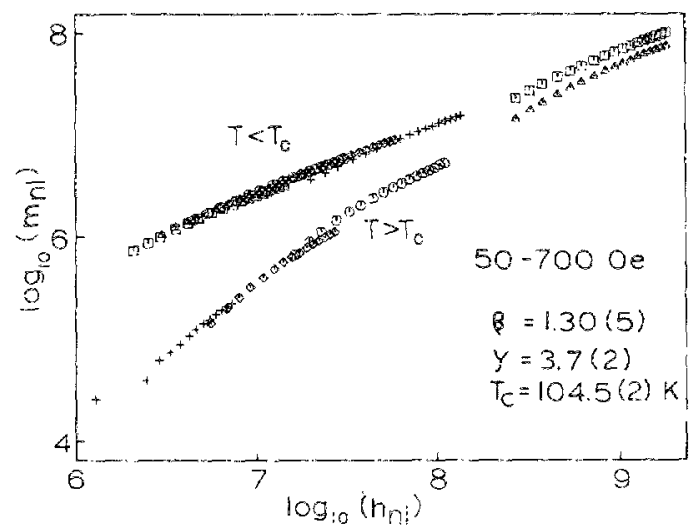

FIG. 4. Nonlinear scaling of the magnetic isotherms for the $x=50$ at. \% Tb glass. $m_{n 1}$ and $h_{21}$ are reduced units as described in the text. The temperatures of the magnetic isotherms in degrees Kelvin are $108.1(+), 106.7$ $(0), 105.8(0), 105.0(\Delta), 104.1(0), 103.3(+), 102.8(0), 102.4(0)$, $102.1(\Delta), 101.7(\square)$. 
TABLE I. Nonlinear critical exponents in RMA systems.

\begin{tabular}{|c|c|c|c|c|c|c|}
\hline System & $\beta$ & $\gamma$ & $\delta$ & $\begin{array}{c}H \text { range } \\
\text { (Oe) }\end{array}$ & $\epsilon$ range & Reference \\
\hline $\operatorname{Dy} \mathbb{N i}_{1.33}$ & $1.2(1)$ & $2.0(1)$ & $2.7(1)$ & $2.4-100$ & $0.005-0.25$ & 7 \\
\hline $\mathrm{Tb}_{64} \mathrm{Fe}_{30} \mathrm{Ga}_{16}$ & $1.7(1)$ & $3.7(1)$ & $3.2(2)^{2}$ & $40.0-288$ & $0.002-0.13$ & 8 \\
\hline $\mathrm{Ge}_{15} \mathrm{~Tb}_{50} \mathrm{Co}_{35}$ & $1.30(5)$ & $3.7(2)$ & $3.8(3)^{\mathrm{a}}$ & $100.0-900$ & $0.005-0.04$ & This work \\
\hline
\end{tabular}

$\delta$ is calculated from the measured critical exponents $\beta$ and $\gamma$ using $\delta=\gamma / \beta+1$. The error in $\delta$ is calculated from a quadrature combination of the errors in $\beta$ and $\gamma$.

state Eq. (1). These magnetic isotherms were taken using a wire wound coil to avoid small hysteresis and remanent field (about $20 \mathrm{Oe}$ ) effects in the superconducting magnet. We find a scaling of the magnetic isotherms according to this equation as shown in Fig. 4. The values of $\beta$ and $\gamma$ determined from this scaing analysis are given in Table $I$ along with those determined for other systems. The low reduced fieid slope of the lower curve corresponding to $T>T_{c}$ in the logarithmic plot of Fig. 4 is close to 2 as expected. However, the crossover to ferromagnetic scaling at high fields apparently does not allow us to get high enough reduced fields to obtain the limiting slope of $2 / \delta[\delta=(\gamma / \beta)+1]$ at high reduced fields.

We believe that although the RMA in this system is strong, the strong exchange allows the system to cross over to standard ferromagnetic behavior in a sufficiently large field. In order to see the nonlinear critical phenomena characteristic of the groundstate of this system, fields below about 700 De must be used to avoid strongly perturbing the speromagnetic groundstate. It has been suggested that systems following nonlinear scaling behavior fall into two main types and the low values of $\gamma$ and $\delta$ in DyNi.133 (Ref. 7) and AuFe (Ref. 12) alloys compared to spin-glass systems without anisotropy (a strong Dzaloshinski-Moriya anisotropy exists in $\mathrm{Au}$-Fe alloys) have been used as evidence in favor of this hypothesis. The exponents of this work and those of $R$ ef. 8 do not, however, follow this pattern. It is likely that diferent classes of systems which follow nonlinear scaling do exist but we do not believe any evidence exists in the measured critical exponents so far for such a classification.
In summary we have shown that alloying $\mathrm{Gd}_{65} \mathrm{CO}_{35}$ with $\mathrm{Tb}$ induces a strong RMA which leads to a crossover from ferrimagnetic to speromagnetic behavior with increasing $x$. A scaling of the magnetic isotherms was found to hold in the alloy with composition $x=50$ at. \% which for high fields followed standard ferromagnetic critical behavior and for fields below about 700 oe followed nonlinear critical behavior. These results show that a ferromagnetic state which shows a standard critical behavior may be induced in an RMA system showing low-field nonlinear critical behavior by application of a large enough magnetic field.

This work was supported in part by the NSF under Grant No. INT-84199546.

13. M. D. Coey, J. Appl. Phys. 49, 1646 (1978).

${ }^{2}$ K. Fukamichi, T. Goto, T. Sakakibara, S. Todo, K. Aoki, and T. Masumoto, J. Magn. Magn. Mater. 54, 239 (1986).

${ }^{3}$ M. J. O'Shea, X. M. Lee, and F. Othman, Phys. Rev. B 34, 4944 (1986). ${ }^{4}$ S. Legvold, in Ferromagnetic Materials, edited by E. P. Wohlfarth (North-Holland, Amsterdam, 1980), Vol. 1, p. 78.

5. J. Rhyne, J. A. Schelleng, and N. C. Koon, Phys. Rey. B 10, 4672 (1974).

${ }^{5}$ D. J. Sellmyer and S. Nafis, J. Appl. Phys. 57, 3584 (1985).

${ }^{7}$ B. Dieny and B. Barbara, Phys. Rev. Lett. 57, 1169 (1986)

${ }^{8}$ D. J. Sellmyer and S. Nafis, Phys. Rev. Lett. 57, 1173 (1986).

${ }^{9}$ P. Beauvillain, C. Ckrappert, and J. P. Renard, J. Phys. Lett. (Paris) 45, L665 (1984) and references therein.

1"S. N. Kaul, J. Magn. Magn. Mater. 53, 5 (1985) and references therein.

"See, for example, M. J. O'Shea and D. J. Sellmyer, Phys. Rev. B 32, 7502 (1985).

${ }^{12}$ P. Monod and H. Bouchiat, J. Phys. (Paris) Lett. 43, L45 (1982), H. Bouchiat, J. Phys. (Paris) \$7, 71 (1986). 